\title{
The correction for the gamma-ray component in neutron therapy
}

\author{
A. M. Kellerer \\ Institut für Medizinische Strahlenkunde der Universität Würzburg, Versbacher Str.5, D-8700 Würzburg, Germany
}

\section{J. Rassow}

Institut für Medizinische Strahlenphysik and Strahlenbiologie, Universitätsklinikum der Gesamthochschule Essen, Hufelandstr.55, D-4300 Essen 1, Germany

(Received 12 October 1979; accepted for publication 4 March 1980)

\begin{abstract}
Neutron beams for therapy always contain some $\gamma$ ray contamination that varies with depth and with distance from the beam axis. The problem therefore arises how the varying $\gamma$ ray contribution should be accounted for in dose specification. Not infrequently a "total effective dose" $D_{E}$ is quoted that is equal to the neutron dose plus the $\gamma$ ray dose divided by a constant weight factor $\tau$. On general biophysical considerations this appears to be not a valid approach since it must be assumed that $\tau$ decreases with increasing dose. The nature and the magnitude of this dose dependence is derived in the present article. Application of the results to actual doses per fraction and to factual $\gamma$ ray to neutron ratios demonstrates that the dose dependence of $\tau$ has, in fact, very minor influence on the numerical values of $D_{E}$. Utilization of a constant value $\tau$ is therefore satisfactory in practice.
\end{abstract}

Key words: neutron therapy, neutron dosimetry, dose specification

\section{INTRODUCTION}

The specification of absorbed dose in a patient subjected to radiation therapy requires data on the spatial distribution of absorbed dose, but must also include information on radiation quality and its spatial variations. ${ }^{1}$ A complete specification would have to be given in terms of the spectral distributions of fluence in particle type and particle energy in all points within the irradiated volume. For neutron beams this can be particularly important, as there may be substantial differences in radiation quality at different distances from the beam axis. ${ }^{2}$ However, in practice, it will frequently be impossible to achieve a complete description and a simplified treatment is therefore not uncommon. In a first approximation the change of radiation quality can be characterized by the varying ratio of neutron to $\gamma$-ray dose, and quoting the two doses separately can therefore be a satisfactory procedure. Frequently one may wish to go even one step further and specify a neutron dose that is equivalent to the combination of the actual neutron and $\gamma$-ray doses. The problem then arises whether it is acceptable to use a constant weight factor for the $\gamma$-ray component or whether it is essential to apply a variable factor that depends on the magnitude of the neutron and the $\gamma$-ray doses. This problem will be considered.

\section{COMBINED EFFECT OF THE NEUTRON AND GAMMA DOSE}

The need for the computation of an effective dose per fraction arises mainly in the consideration of effects on the normal tissue where tolerance levels are commonly related to a single quantity. An example of such a quantity is the nominal dose, computed by the Ellis-formula or its modification for neutron irradiation. ${ }^{3}$ The derivation of this or similar quantities requires the statement of effective doses per fraction rather than the separate specification of a neutron and a $\gamma$-ray dose. The following considerations will therefore refer to normal tissue rather than to the tumor. This simplifies the discussion because the complicating aspect of hypoxic cells and of reoxygenation need not be taken into account. Instead the analysis can be linked to a consideration of survival curves. Survival curves of mammalian cells can be represented by various equations. Among these the linear-quadratic equation for the logarithm of the survival $S$

$$
-\ln S=a D+(b D)^{2}
$$

is of the simplest form and it has the advantage that it permits a straightforward biophysical interpretation. ${ }^{4}$ In this interpretation the linear term corresponds to a one-particle effect. The quadratic term $(b D)^{2}$ corresponds to a two-particle effect. As the coefficients $a$ and $b$ depend, among other factors on radiation quality, one has different survival relations for neutrons and for $\gamma$ rays:

$$
\begin{aligned}
& -\ln S_{N}=a_{N} D_{N}+\left(b_{N} D_{N}\right)^{2} \\
& -\ln S_{G}=a_{G} D_{G}+\left(b_{G} D_{G}\right)^{2} .
\end{aligned}
$$

The indices $N$ and $G$ refer to neutrons and to $\gamma$ rays. According to the biophysical interpretation ${ }^{4}$ the logarithmic reduction of cell survival probability, $-\ln S$, is proportional to the yield of lesions. The linear terms in absorbed dose represent lesions that are formed in individual particle tracks. These linear terms are therefore simply additive for neutrons and $\gamma$ rays. The quadratic terms represent the pairwise interaction of sublesions that are formed by separate 
particles; they are therefore proportional to the square of the yield of sublesions. It follows that the yield of sublesions is for $\gamma$ rays proportional to $b_{G} D_{G}$ and for neutrons to $b_{N} D_{N}$. Accordingly the quadratic term for the mixed field is the square of the term $\left(b_{G} D_{G}+b_{N} D_{N}\right)$ that represents the yield of sublesions. One obtains consequently the following relation for a $\gamma$-ray dose $D_{G}$ and a neutron dose $D_{N}$ applied in one session:

$$
\begin{aligned}
&-\ln S_{N+G}=a_{N} D_{N}+a_{G} D_{G}+\left(b_{N} D_{N}+b_{G} D_{G}\right)^{2} \\
&=a_{N} D_{N}+a_{G} D_{G}+\left(b_{N} D_{N}\right)^{2}+\left(b_{G} D_{G}\right)^{2} \\
&+2 b_{N} b_{G} D_{N} D_{G} .
\end{aligned}
$$

Zaider and $\operatorname{Rossi}^{5}$ give a more detailed discussion of this survival equation under a mixed irradiation. It should be pointed out that the linear-quadratic relation may be merely an approximation that is in reasonable agreement with experimental data without being rigorously valid. It can nevertheless be utilized, since the present analysis aims not at a precise formulation but at a rough assessment of the nature of the dose dependence for the weight factor $\tau$.

\section{DETERMINATION OF THE WEIGHT FACTOR}

Equation (3) can be used to compute the neutron dose $D_{E}$ that has an effect equal to that of the actual neutron and the $\gamma$-ray dose combined. This dose, $D_{E}$, will be called the total effective dose (the notation employed in this article follows the conventions proposed by ECNEU ( $E$ uropean Clinical Neutron Dosimetry Group) in the fourth draft of a European Protocol for Neutron Dosimetry for External Beam Therapy (August 1979). $D_{\mathrm{N}}$ : absorbed dose of neutrons; $\mathrm{D}_{\mathrm{G}}$ : absorbed dose of $\gamma$ rays; $D_{T}$ : total absorbed dose; $D_{E}=$ total effective dose $\left.=D_{N}+D_{G} / \tau\right)$ of a neutron beam contaminated by $\gamma$ rays. The weight factor for the neutron relative to the $\gamma$-ray dose in the computation of the equivalent neutron dose $D_{E}$ will be designated by $\tau$ :

$$
D_{E}=D_{N}+D_{G} / \tau
$$

From the condition:

$$
\begin{aligned}
a_{N} D_{E}+b_{N}^{2} D_{E}^{2}=a_{N} D_{N}+a_{G} D_{G}+b_{N}^{2} D_{N}^{2} \\
+b_{G}^{2} D_{G}^{2}+2 b_{N} b_{G} D_{N} D_{G}
\end{aligned}
$$

one obtains:

$$
\begin{gathered}
a_{N} D_{N}+a_{N} D_{G} / \tau+b_{N}^{2} D_{N}^{2}+2 b_{N}^{2} D_{N} D_{G} / \tau+b_{N}^{2} D_{G}^{2} / \tau^{2} \\
=a_{N} D_{N}+a_{G} D_{G}+b_{N}^{2} D_{N}^{2}+b_{G}^{2} D_{G}^{2}+2 b_{N} b_{G} D_{N} D_{G}
\end{gathered}
$$

and:

$$
\begin{aligned}
\tau^{2}\left(a_{G}+b_{G}^{2} D_{G}+2 b_{N} b_{G} D_{N}\right) \\
-\tau\left(a_{N}+2 b_{N}^{2} D_{N}\right)-b_{N}^{2} D_{G}=0 ;
\end{aligned}
$$

therefore:

$$
\tau=\frac{\left(c_{2}^{2}+4 c_{1} c_{3}\right)^{1 / 2}+c_{2}}{2 c_{1}}
$$

with:

$$
\begin{gathered}
c_{1}=a_{G}+b_{G}^{2} \rho D_{T}+2 b_{N} b_{G}(1-\rho) D_{T} \\
c_{2}=a_{N}+2 b_{N}^{2}(1-\rho) D_{T} \\
c_{3}=b_{N}^{2} \rho D_{T}
\end{gathered}
$$

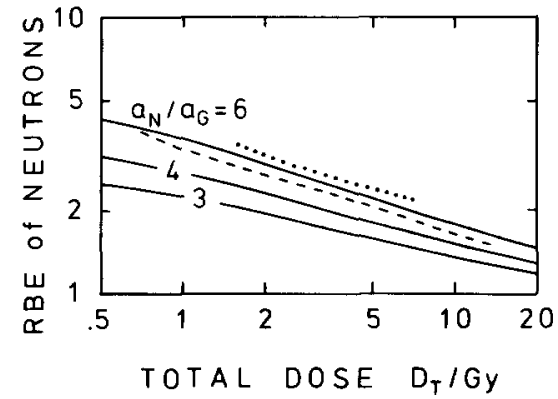

FIG. 1. RBE of neutrons versus $\gamma$ rays for the linear-quadratic relation [Eq (2)] and the parameters $b_{N}=b_{G}=0.16 \mathrm{~Gy}^{-1}, a_{G}=0.13 \mathrm{~Gy}^{-1}$, and $a_{N} / a_{G}$ $=6,4$, or 3 (solid lines). The broken line corresponds to a curve derived by Field (1) for $d(15) \mathrm{Be}$-neutrons and various effects on the skin; the dotted line corresponds to a curve given by Field (1) for acute effects on the intestine.

where $D_{T}$ is the total absorbed dose:

$$
D_{T}=D_{N}+D_{G},
$$

and $\rho$ the relative $\gamma$ ray contribution:

$$
\rho=D_{G} / D_{T} .
$$

The formula for $\tau$ appears somewhat complicated, but it is readily evaluated. It will be noted that at small doses the value $\tau$ converges towards $a_{N} / a_{G}$, while for large doses it tends towards $b_{N} / b_{G}$. If the $\gamma$-ray dose is small compared to the neutron dose $(\rho \ll 1)$ one obtains the simpler relation:

$$
\tau \approx \frac{a_{N}+2 b_{N}^{2} D_{T}}{a_{G}+2 b_{N} b_{G} D_{T}} .
$$

\section{NUMERICAL EVALUATION AND DISCUSSION}

Hall ${ }^{6,7}$ has recently performed extensive intercomparisons of survival curves of V-79 hamster cells exposed to neutron beams at various therapy installations. The experiments include also repeated control experiments with $\gamma$ rays.

The $\gamma$-ray survival curves can be represented by the parameters $a_{G}=0.13 \mathrm{~Gy}^{-1}$ and $b_{G}=0.16 \mathrm{~Gy}^{-1}$. The neutron survival curves do not permit the derivation of precise values of $b_{N}$. The results of earlier experiments ${ }^{8,9}$ are also somewhat uncertain with regard to $b_{N}$. At neutron energies below 1 $\mathrm{MeV}$ there is evidence that $b_{N}$ exceeds $b_{G}$; for larger energies, as applied in therapy, any difference between $b_{N}$ and $b_{G}$ appears to be small. Results will therefore first be derived for an assumed equality of the coefficients $b_{N}$ and $b_{G}$; subsequently, larger values for $b_{N}$ will be considered and it will be seen that the dependence of $\tau$ on dose will then be less important than for equal values $b_{N}$ and $b_{G}$.

Estimated values of $a_{N}$ vary from $a_{N}=0.46 \mathrm{~Gy}^{-1}$ for a neutron beam produced by $d(50)+$ Be neutrons (Tamvec) to $a_{N}=0.66 \mathrm{~Gy}^{-1}$ for $d(15)+$ Be neutrons (Hammersmith). The ratios $a_{N} / a_{G}$ are therefore contained within a range of values from roughly $3-6$, and the dependence of RBE on the total dose $D_{T}$ that corresponds to the values 3,4 , and 6 is plotted in Fig. 1. A comparison to in vivo data indicates substantial agreement with this dependence between RBE and dose. Field ${ }^{3}$ has compiled results from various authors for the RBE of $d(15)+$ Be neutrons for skin damage. In Fig. 


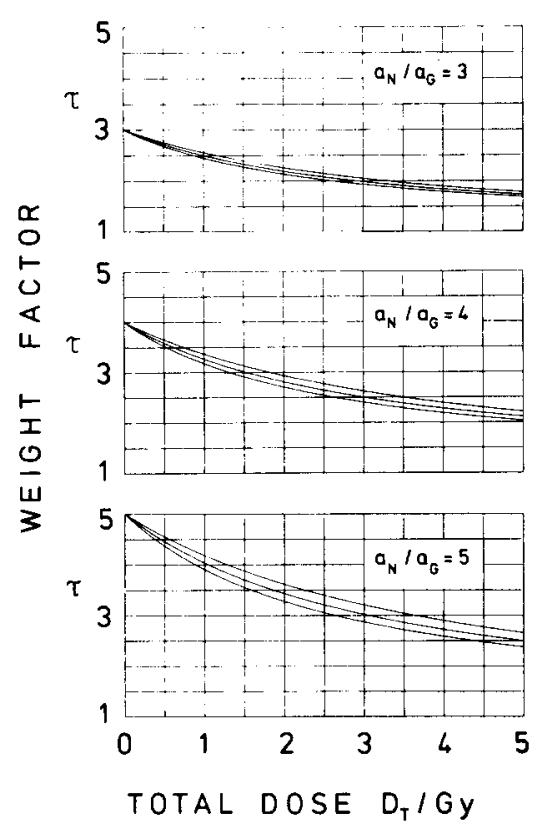

FIG. 2. Weight factor $\tau$ as a function of total dose $D_{T}$. $\rho$ is the ratio of the $\gamma$-ray dose to the total dose. The three curves belong (beginning from the lowest curve) to the values $p=0.1,0.5$, and 0.9 . The parameters [see Eq. (2)] are $b_{N}=b_{G}=0.16 \mathrm{~Gy}^{-1}$ and $a_{G}=0.13 \mathrm{~Gy}^{-1}$. The panels belong to the ratios $a_{N} / a_{G}=5,4$, and 3 .

1 his relation for the RBE as a function of neutron dose per fraction is replotted (broken line). A second curve (dotted line) is a replot of the curve given by Field for acute intestinal damage. Apart from possible differences at small doses, there is reasonable agreement between the in vitro and in vivo results, as far as the characteristic dependence of RBE on dose is concerned. This supports the utilization of the in vitro data, i.e., of the survival curve parameters. Accordingly the factor $\tau$ is calculated with the values $a_{G}=0.13 \mathrm{~Gy}^{-1}$ and $b=b_{N}$ $=b_{G}=0.16 \mathrm{~Gy}^{-1}$ and with various ratios of the neutron $\mathrm{RBE}$ at low doses, $a_{N} / a_{G}$.

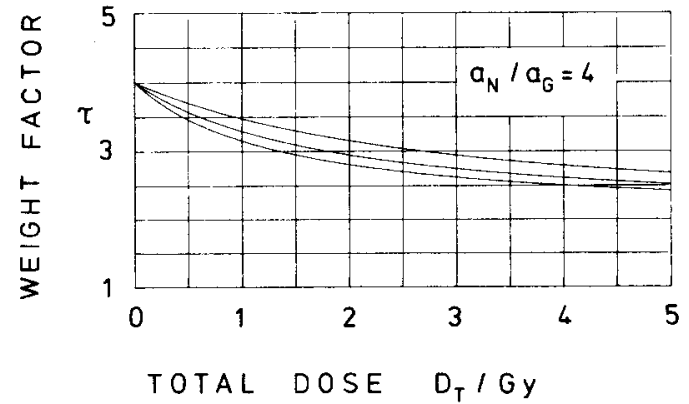

Fig. 4. Weight factor $\tau$ as a function of total dose $D_{T}$. $\rho$ is the ratio of the $\gamma$-ray dose to the total dose. The three curves belong (beginning from the lowest curve) to the values $\rho=0.1,0.5$, and 0.9 . The parameters [see Eq. (2)] are $b_{N}=2 b_{G} ; b_{G}=0.16 \mathrm{~Gy}^{-1} ; a_{N} / a_{G}=4$, and $a_{G}=0.13 \mathrm{~Gy}^{-1}$.

This will make it possible to derive the dependence of $\tau$ on dose. The nature of this dependence will not critically depend on the exact values of $a_{G}$ and $b$; the results are therefore meaningful even if the application of data from cell culture experiments to the reaction of the irradiated tissue is doubtful. The results are given in Fig. 2. The different panels refer to the values 3,4 , and 5 of the ratio $a_{N} / a_{G}$. In each graph curves are given for $\rho=0.1,0.5$, and 0.9 . The values for $\rho=0.1$ are nearly identical to the values for $\rho \approx 0$ that result from Eq. (12).

At doses $D_{T}$ of $1-2 \mathrm{~Gy}$ the graph for $a_{N} / a_{G}=4$ agrees well with the value of $\tau=3$ that is presently used at most neutron therapy centers for $d(15)+$ Be neutrons. It provides therefore the dependence of values $\tau$ on total dose that can be compared to the use of a fixed value $\tau$.

Table 1 exemplifies the differences in numerical values for the effective dose $D_{E}$ that result from the use of fixed and variable values $\tau$. The ratios of the $\gamma$ ray to neutron dose for two depths at the isocentric neutron therapy facility CIRCE in Essen (see Fig. 3) are used in the comparison. Two current fractionation schemes (Hammersmith ${ }^{10,11}$, and Essen ${ }^{12,13}$ )

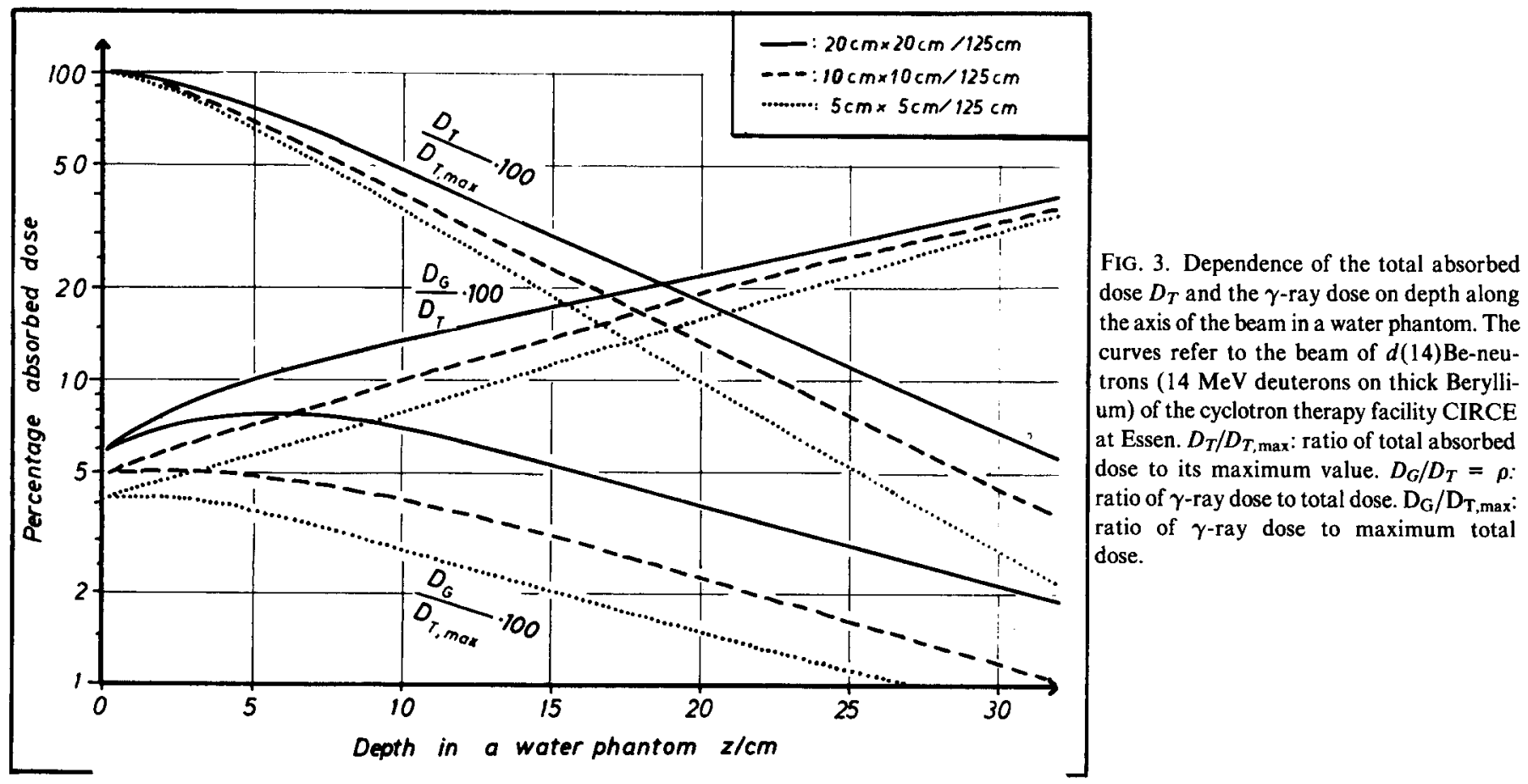


TABLE 1. Practical consequences of using variable weight factors $\tau$ for $d(14)+$ Be neutrons (mean energy $5.5 \mathrm{MeV}$ ) of the cyclotron neutron therapy facility CIRCE in Essen, Field size $20 \mathrm{~cm} \times 20 \mathrm{~cm}$; source-surface-distance $125 \mathrm{~cm}$; water phantom.

\begin{tabular}{|c|c|c|c|c|}
\hline \multirow[b]{3}{*}{ Parameters for single fraction } & \multicolumn{2}{|c|}{$\begin{array}{l}\text { Fractionation } \\
\text { Hammersmith }\end{array}$} & \multicolumn{2}{|c|}{$\begin{array}{c}\text { Fractionation } \\
\text { Essen }\end{array}$} \\
\hline & \multicolumn{4}{|c|}{ Phantom depth } \\
\hline & $\begin{array}{c}\mathrm{z}_{0}=5 \\
\mathrm{~cm}\end{array}$ & $\begin{array}{l}\mathrm{z}_{1}=20 \\
\mathrm{~cm}\end{array}$ & $\begin{array}{c}\mathrm{z}_{0}=5 \\
\mathrm{~cm}\end{array}$ & $\begin{array}{c}\mathrm{z}_{1}=20 \\
\mathrm{~cm}\end{array}$ \\
\hline$D_{N} / \mathrm{Gy}$ & 1.300 & 0.271 & 0.772 & 0.161 \\
\hline$D_{G} / G y$ & 0.144 & 0.074 & 0.086 & 0.044 \\
\hline$D_{T} / \mathrm{Gy}$ & 1.444 & 0.345 & 0.856 & 0.205 \\
\hline$\rho=D_{G} / D_{T}$ & 0.100 & 0.214 & 0.100 & 0.214 \\
\hline$D_{T}\left(z_{1}\right) / D_{T}\left(z_{0}\right)$ & \multicolumn{2}{|c|}{0.239} & \multicolumn{2}{|c|}{0.239} \\
\hline \multicolumn{5}{|l|}{$\begin{array}{l}\text { Commonly used constant } \\
\text { weight factor }\end{array}$} \\
\hline$\tau$ & 3.00 & 3.00 & 3.00 & 3.00 \\
\hline$D_{E} / \mathrm{Gy}$ & 1.348 & 0.296 & 0.800 & 0.176 \\
\hline$D_{E}\left(z_{1}\right) / D_{E}\left(z_{0}\right)$ & \multicolumn{2}{|c|}{0.220} & \multicolumn{2}{|c|}{0.220} \\
\hline \multicolumn{5}{|l|}{$\begin{array}{l}\text { Variable weight factor } \tau \text { for } \\
a_{N} / a_{G}=0.52 / 0.13=4 \\
\text { according Fig. } 2\end{array}$} \\
\hline$\tau$ (approx. Eq. 12) & 2.91 & 3.64 & 3.24 & 3.78 \\
\hline$\tau(\mathrm{Eq} .8,9)$ & 2.94 & 3.67 & 3.27 & 3.80 \\
\hline$D_{E} / \mathrm{Gy}$ & 1.349 & 0.291 & 0.798 & 0.173 \\
\hline$D_{E}\left(z_{1}\right) / D_{E}\left(z_{0}\right)$ & \multicolumn{2}{|c|}{0.216} & \multicolumn{2}{|c|}{0.217} \\
\hline \multicolumn{5}{|l|}{$\begin{array}{l}\text { Variable weight factor } \tau \text { for } \\
a_{N} / a_{G}=0.66 / 0.13 \approx 5 \\
\text { according Fig. } 2\end{array}$} \\
\hline$\tau$ [approx. Eq. (12)] & 3.60 & 4.59 & 4.05 & 4.77 \\
\hline$\tau$ Eqs. (8), (9) & 3.63 & 4.63 & 4.08 & 4.80 \\
\hline$D_{E} / \mathrm{Gy}$ & 1.340 & 0.287 & 0.793 & 0.170 \\
\hline$D_{E}\left(z_{1}\right) / D_{E}\left(z_{0}\right)$ & \multicolumn{2}{|c|}{0.214} & \multicolumn{2}{|c|}{0.215} \\
\hline
\end{tabular}

are considered. (The fractionation on the basis of total effective dose, $D_{E}$, has been agreed upon by three members (Amsterdam, Edinburgh, Essen) of the EORTC Project Group on Neutron and Fast Particle Therapy.)

The computations are performed according to Eqs. 8 and 9 or the approximation of Eq. 12 with the parameters $a_{G}=$ $0.13 \mathrm{~Gy}^{-1}, b_{G}=b_{N}=0.16 \mathrm{~Gy}^{-1}$, and the value $a_{N}=0.52$ $\mathrm{Gy}^{-1}$ that agrees with the ratio $a_{N} / a_{G}=4$ (intermediate panel in Fig. 2). The results are given in the penultimate row of boxes in Table 1 . At the bottom of Table 1 numerical results are given for the value $a_{N}=0.66 \mathrm{~Gy}^{-1}$ that corresponds to the lower panel of Fig. 2 and that is indicated by the cell culture experiments of $\mathrm{Hall}^{6,7}$ with the $d(15)+$ Be neutrons of Hammersmith.

The essential result is that there is, indeed, a pronounced dependence of the weight factor $\tau$ on total dose $D_{T}$. This applies for both sets of parameters that are considered. However, neither the absolute uncertainty of $\tau$ nor its dependence on $D_{T}$ have substantial influence on the magnitude of the resulting effective dose $D_{E}$. The use of a fixed weight factor $\tau$ is therefore still justified in clinical applications.

It remains to examine the possibility that the coefficient of the quadratic term in absorbed dose is larger for neutrons than for $\gamma$ rays. For this purpose the weight factor $\tau$ is derived for $a_{N} / a_{G}=4$ as in Fig. 2, but with $b_{N} / b_{G}=2$. The results are given in Fig. 4. As expected, the dependence of $\tau$ on total dose is less pronounced in this case. This implies that the use of a constant weight factor $\tau$ leads to even smaller imprecisions if $b_{N}$ exceeds $b_{G}$.

\section{CONCLUSION}

In agreement with experimental observations and with general biophysical considerations, the RBE of neutrons relative to $\gamma$. rays is largest at smallest doses. It follows that the factor $\tau$, that weights the neutron absorbed dose relative to the $\gamma$ ray absorbed dose, decreases with increasing dose per fraction. From the linear-quadratic dependence of the logarithm of cellular survival and from parameters of survival curves, a numerical dependence of $\tau$ on total dose can be obtained.

Although the dependence of $\tau$ on dose is pronounced, it plays only a very minor role in actual numerical evaluations. It is consequently acceptable to apply a fixed weight factor $\tau$ in order to correct for the $\gamma$-ray component in a neutron beam. In the present analysis parameters from cell survival curves are used as input data to obtain general guidance on the magnitude of the numerical values in the equations. However, it is evident that appropriate values of $\tau$ must be based on radiobiological investigations and clinical trials with a particular neutron beam.

The general conclusion of this investigation will not be applicable to a mixed modality treatment where substantial $\gamma$ ray and neutron doses are given in one session. Only in such cases would the dose dependence of $\tau$ have to be taken into account.

${ }^{1}$ ICRU, Report 29, "Dose Specification for Reporting External Beam Therapy with Photons and Electrons", International Commission on Radiation Units and Measurements (ICRU, Washington, 1978).

${ }^{2}$ H. G. Menzel, A. J. Wacker, R. Grillmaier, and L. Bihi, "Radiation Quality Studies in Mixed Neutron-Gamma Fields" in Proceedings of the Third Symposium on Neutron Dosimetry 1977, edited by G. Burger and H. G. Ebert (EURATOM, EUR 5848 DE/EN/FR), 1978, pp. 481497.

${ }^{3}$ S. B. Field, Curr. Top. in Radiat. Res. Q. 11, 1 (1976).

${ }^{4}$ A. M. Kellerer and H. H. Rossi, Curr. Top. in Radiat. Res. Q. 8, 85 (1972).

${ }^{5} \mathrm{M}$. Zaider and H. H. Rossi, Radiat. Res. (in press).

${ }^{6}$ E. J. Hall, Int. J. Radiat. Oncology Biol. Phys 3, 195 (1977).

${ }^{7}$ E. J. Hall and A. M. Kellerer, "Review of RBE Data for Cells in Culture," Proceedings 3rd Meeting on Fundamental and Practical Aspects of the Application of Fast Neutrons and Other High LET Particles in Clinical Radiotherapy (Pergamon Press, Oxford, 1978), pp. 171-174.

${ }^{8}$ E. J. Hall, J. K. Novak, A. M. Kellerer, H. H. Rossi, St. Marino, L. J. Goodman, Radiat. Res. 64, 245 (1975).

${ }^{9}$ A. M. Kellerer, E. J. Hall, H. H. Rossi, and P. Teedla, Radiat. Res. 65, 172 (1976).

${ }^{10} \mathrm{M}$. Catterall, D. K. Bewley, and J. Sutherland, Brit. Med. J. 1642 (1977).

${ }^{11}$ W. Duncan, Brit. J. Radiol. 51, 943 (1978).

12J. J. Battermann, Radiol. Clin. 47, 464 (1978).

${ }^{13}$ G. Schmitt, E. Scherer, Strahlentherapie 157, 851 (1979). 